\title{
TIEMPO Y NATURALEZA DE USO DEL MÓVIL EN ESTUDIANTES DE LA CARRERA DE MEDICINA.
}

\author{
Time and Nature of Mobile Phone Usage Medicine Students. \\ *Lourdes Azucena Andrade Avila, ** José A. Ortega, ** José M. Santos, **Luis F. Rivas, \\ ** Néstor R. Banegas, **Sofía M. Gámez.
}

\section{RESUMEN}

Antecedentes: Diversas investigaciones se realizan sobre el tiempo y naturaleza de uso del móvil en estudiantes universitarios pero escasas en el área de la medicina en el siglo de la tecnología. Objetivo: Establecer el tiempo y naturaleza de uso del teléfono móvil en estudiantes de cuarto año de medicina de cuarto año A en la Escuela Universitaria de Ciencias de la Salud de la Universidad Nacional Autónoma de Honduras en el Valle de Sula (EUCS/UNAH-VS) en el primer semestre académico del 2017. Pacientes y Métodos: Estudio exploratorio-descriptivo. Población fue de 78, muestra no probabilística por conveniencia de 38 estudiantes, instrumento de recolección de datos se realizó a través del programa App Usage ${ }^{\circledR}$ que generó los resultados, se evaluaron los datos de frecuencias, porcentajes y medidas de tendencia central en Excel ${ }^{\circledR}$. Resultados: Los datos reflejaron que el tiempo promedio de uso del móvil por los estudiantes fue de 5 horas 9 minutos $(21.5 \%)$ del día. El uso se centró en aplicaciones móviles reactivas (distractor) con 4 horas 47 minutos (93\%) y en aplicaciones móviles proactivas solo 22 minutos $(7 \%)$. Conclusiones: Los datos de esta investigación indican que el patrón de uso del teléfono móvil es del $21.5 \%$ al día y la naturaleza del uso de los teléfonos celulares por parte del estudiante de Medicina de cuarto año A de la UNAH-VS es de carácter reactivo o sea como distractor.

\footnotetext{
* Especialista en Anestesiología / Docente de la Escuela Universitaria de Ciencias de la Salud de la Universidad Nacional Autónoma de Honduras en el Valle de Sula ( EUCS/UNAH-VS)

**Estudiantes de 4 año de Medicina de la EUCS/UNAH-VS

Dirigir correspondencia a joseortega7@gmail.com

Recibido: 10 de julio del 2017 Aprobado: 01 de mayo del 2018
}

\section{PALABRAS CLAVE}

Aplicaciones Móviles, estudiantes de medicina, Teléfonos celulares.

\begin{abstract}
Background: Various researches are carried out on the time and nature of cell phone use in university students, but scarce in the field of medice in the century technology. Objetive: To establish the time and nature of the use of mobile phone in fourth year A student of medicine at the Escuela Universitaria de Ciencias de la Salud de la Universidad Nacional Autonoma de Honduras en el Valle de Sula (EUCS/UNAH-VS) in the first semestre of 2017. Patients and Methods: Exploratory descriptive study. Population: 78, sample by consent of 38 student, instrumet it was done through the App Usage ${ }^{\circledR}$ program that generated the results, the data of frequencies, percentages and measures of central tendency in Excel ${ }^{\circledR}$ were evaluated. Results: The data showed that the average time of student use of mobile was 5 hours with 9 minutes a day (21.5\%). The use focused on reactive mobile application (distractor) with 4 hours 47 minutes $(93 \%)$ and in proactive mobile applications only 22 minutes (7\%). Conclusions: The data of this research indicate that the pattern of use of mobile phone is of $21.5 \%$ in a day, and the nature of the use of cell phone by fourth year medical student a of the EUCS/UNAH-VS is of a character reactive or its like distractor.
\end{abstract}

\section{KEYWORDS}

Medical students, Cell phone, Mobile application. 


\section{INTRODUCCIÓN}

El uso de las Tecnologías de la Información y de la Comunicación (TIC) está creciendo a un ritmo acelerado y está dando lugar a cambios importantes en la sociedad, permitiendo que la información esté al alcance de todos y ofreciendo nuevos canales de comunicación y de entretenimiento. Estas herramientas introducen nuevas vías de relación entre personas capaces de mejorar su calidad de vida y que resultan relevantes en la empresa, la docencia o la sanidad, entre otros.

El teléfono móvil, gracias a su conexión a internet (Smartphone), ha pasado a ser un objeto donde se almacena todo tipo de información y se dispone de múltiples aplicaciones que dan respuesta a los gustos y necesidades de su propietario. Por lo tanto, internet hizo que su funcionalidad sea muy amplia. Los mayores consumidores de las TIC son los jóvenes. ${ }^{(1)}$

Los recursos tecnológicos han modificado la forma de comunicación en las aulas. Existen diferentes metodologías (Flipped Classroom, Blended-Learning, entre otras) que usan distintos tipos de dispositivos móviles (teléfonos o tabletas). Estos dispositivos podemos utilizarlos junto con internet para dar una mayor respuesta a la gran variedad de aplicaciones existentes, para permitir el acceso a los recursos educativos necesarios como son archivos, enlaces web o plataformas Moodle, y para facilitar la información a aquellos estudiantes que tengan dificultades en la movilidad. Otra ventaja de los dispositivos digitales está en su uso como herramienta en el trabajo activo-colaborativo, permitiéndoles el desarrollo de la competencia digital o la competencia interpersonal a través del trabajo colaborativo. ${ }^{(2)}$

La formación académica del estudiante de medicina implica una serie de ejercicios y prácticas históricas, que no cambian a través del tiempo, lo único que cambia son sus actores. El estudiante de medi- cina es sin duda un estudioso por excelencia por sus años de estudio, por el compromiso curricular y por la obligación ética y moral en pro de sus futuros pacientes. ${ }^{(3)} \mathrm{El}$ advenimiento de la tecnología y en particular el realce mundial de la industria de telecomunicaciones, ha impactado a la sociedad, ya que se han vuelto usuarios "crónicos" de éstas, en especial del teléfono móvil. Los impactantes resultados de investigaciones en otros países sobre la naturaleza del uso del teléfono móvil, ${ }^{(4)}$ despertaron en este grupo investigador el deseo de conocer el tiempo y la naturaleza que los estudiantes de medicina le dan al uso del teléfono móvil.

Historia del teléfono móvil: En 1876, "Alexander Graham Bell patentó el teléfono para que Marconi años más tarde resolviera la forma de transmisión de señal del mensaje. Casi cinco décadas transcurrieron para que el desarrollo del teléfono móvil tomara el rumbo deseado. Desde 1950, los laboratorios Bell, que operaban en Estados Unidos, entendieron el concepto de celular $y$ mediante sus investigaciones lograron desarrollar la primera patente aceptada en 1960, tiempo en el cual Cooper y su equipo de Motorola presentaron el primer prototipo de teléfono móvil. Un año después, la alianza de AT\& ${ }^{\circledR}$ (American Telephone and Telegraph $\left.^{\circledR}\right)$, y Laboratorios Bell ${ }^{\circledR}$ dejó como resultado la integración del móvil a una red de comunicación". ${ }^{(5)}$

Historia y uso del teléfono móvil en Honduras: Fue en la administración del presidente Carlos Roberto Reina que se otorgó un contrato a la compañía Lucent Technologies de $A T \& T^{\circledR}$ para instalar el sistema AMPS (Advanced Mobile Phone System) en el corredor de Tegucigalpa y San Pedro Sula. ${ }^{(6)}$ Según datos de CONATEL (Comisión Nacional de Telecomunicaciones) las líneas telefónicas móviles alcanzaron un total de abonados de 7,717,200 al finalizar el tercer trimestre de 2016. Es decir, en cuanto a densidad telefónica móvil, el número de 
líneas telefónicas móviles por cada 100 habitantes, alcanzó un valor de 88.86, lo que representa que 89 de cada 100 hondureños posee una línea telefónica móvil. ${ }^{(6)}$

En cuanto al acceso de Internet, CONATEL refleja que el número de usuarios de Internet por cada 100 habitantes, alcanzó un valor de 29.5 durante el año 2016, lo que representa que 30 de cada 100 hondureños acceden a Internet. ${ }^{(7)}$

Naturaleza del uso del teléfono móvil y redes sociales: El uso de aplicaciones móviles es un fenómeno nuevo. Una red social virtual es una estructura social de relaciones entre usuarios a través de Internet en sitios basados en la web, que permiten a los usuarios compartir contenidos, interactuar y crear comunidades sobre intereses similares. ${ }^{(8)}$

Adicción al teléfono móvil: En líneas generales, podemos clasificar el patrón de uso del teléfono móvil en tres grandes categorías en función de la intensidad, frecuencia o cantidad de dinero invertida en ella y del grado de interferencia en las relaciones familiares, sociales y/o laborales de las personas implicadas:

a) Uso no problemático,

b) Uso problemático-abuso y

c) Uso patológico-dependencia. ${ }^{(8-9)}$

Impacto del teléfono móvil y las redes sociales en el rendimiento académico: El uso de celulares se ha incrementado en la población en comparación con la década anterior debido a la gran utilidad que se le puede dar, como una comunicación más fluida. ${ }^{(10)}$

De acuerdo con un estudio realizado en estudiantes de odontología del "College of Dental Sciences and Research", el patrón de utilización de los teléfonos móviles mostró una grave alarma, indicando que los estudiantes habían abusado del tiempo de utilización de sus dispositivos, afectando su desempeño académico de una forma negativa. Así mismo se observó que el $24.7 \%$ de su población estudiada, utilizaba constantemente el móvil durante clases o durante procedimientos prácticos laborales. ${ }^{(11)}$

\section{Efectos del teléfono móvil en el aula de} clases: El móvil se vuelve un distractor, ya que la persona que está al tanto del celular pierde atención desaprovechando así el proceso de aprendizaje, pero además no únicamente el usuario es el afectado, sino que también los que se encuentran cercanos a esa persona pierden la atención, al punto que se contagian y empiezan a hacer uso de su móvil. ${ }^{(12)}$

\section{Desventajas de las redes sociales:}

a) Son peligrosas si no se configura la privacidad correctamente,

b) No hay control de los datos suministrados,

c) Adicción a las redes sociales,

d) No hay control sobre lo que se sube a las redes sociales. ${ }^{(12)}$

La carrera de medicina tiene una malla curricular extensa que implica horario académico de hasta 8 horas en las ciencias básicas. ${ }^{(3)}$ Por su carga académica, cuarto año es considerado, en la EUCS/UNAH-VS, como un año de alta exigencia, en el cual el estudiante dispone de un reducido tiempo de estudio (necesario para la aprobación de las materias y su formación profesional) al salir de sus clases diarias. Tiempo que deberá utilizar eficientemente para lograr el éxito curricular. Existen investigaciones previas que categorizan el tiempo libre del estudiante de medicina al terminar su jornada académica, e identifican el uso del teléfono móvil como un potencial distractor de sus responsabilidades académicas. ${ }^{(13)}$

App Usage ${ }^{\circledR}$ es una aplicación para la plataforma móvil Android ${ }^{\circledR}$, específicamente 
versiones 4.1 y posteriores, desarrolllada por Azsoft Technology ${ }^{\circledR}$, la cual ofrece las siguientes funciones claves para esta investigación: recoleccion del historial de uso de las aplicaciones (nombre y tiempo de uso de cada aplicación), historial de consultas (las veces que el teléfono es consultado), registro de horas a las que se consultan las aplicaciones y cuáles son las más utilizadas. Es de notar que App Usage ${ }^{\circledR}$ unicamente es capaz de recolectar datos mientras está activa. En consecuencia, para evitar que ésta sea cerrada por mecanismos de ahorro de memoria RAM del sistema operativo Android $^{\circledR}$, la misma se mantiene abierta a través de una notificación permanente en la barra de notificaciones. ${ }^{(14)}$

Esta investigación se hizó con el fin de obtener datos concluyentes que reflejen el uso del teléfono móvil de forma proactiva o reactiva por parte de los estudiantes. Se entiende, que si se utiliza el teléfono móvil como herramienta para el estudio, el uso se catalogaría como proactivo y en contraposición, si se utiliza como un distractor, por su característica perjudicial, el uso se enmarcaría en reactivo.

El objetivo de la investigación fue establecer el tiempo y naturaleza de uso del teléfono móvil en estudiantes de medicina de cuarto año $A$ en la EUCS/UNAH-VS en el primer semestre académico del 2017.

\section{MATERIALES Y MÉTODOS}

Se realizó un estudio con alcance exploratorio-descriptivo, analizando la base de datos creada por el grupo investigador y la información obtenida a través de la aplicación móvil para sistema operativo Android ${ }^{\circledR}$, “App Usage $^{\circledR ”}$. La población eran 78 estudiantes de cuarto año A pero con los criterios de inclusión quedo una muestra no probabilística de 38 estudiantes de la carrera de medicina en la EUCS/UNAH-VS en el primer semestre académico 2017.
La participación en este estudio fue delimitada por los siguientes criterios de inclusión:

a) Estar de acuerdo con los criterios de aplicación del estudio, detalladas en el consentimiento informado y la ficha informativa.

b) Estar matriculado en las cuatro asignaturas que corresponden al primer semestre de cuarto año A (Introducción a la Clínica I (CL-100), Fisiopatología I (FP-211), Patología I (PA-100) y Farmacología | (PG-201) de la carrera de medicina en la UNAH-VS.

c) Poseer un teléfono celular (móvil) de uso personal, con el sistema operativo "Android".

d) Tener acceso a Internet con su teléfono.

e) Tener instalada la aplicación App Usage.

La participación en este estudio fue delimitada por los siguientes criterios de exclusión:

a) No estar de acuerdo con los criterios de aplicación del estudio, detalladas en el consentimiento informado y la ficha informativa.

b) No estar matriculado en las cuatro asignaturas que corresponden al primer semestre de cuarto año A (Introducción a la Clínica I (CL-100), Fisiopatología I (FP-211), Patología I (PA-100) y Farmacología I (PG-100)) de la carrera de Medicina en la UNAH-VS.

c) No poseer un teléfono celular (móvil) de uso personal, con el sistema operativo "Android".

d) No tener acceso a Internet durante el tiempo que dure la recolección de datos de la investigación.

e) No tener instalada la aplicación de App Usage $^{\circledR}$.

El proyecto de investigación estuvo compuesto de dos fases. La primera comprendió la recolección de datos a través de la instalación en los teléfonos móviles de los participantes, de una aplicación móvil, "App Usage ${ }^{\circledR}$, la cual recogió información 
sobre el tiempo y naturaleza del uso del teléfono móvil y la envió a través de correo electrónico desglosada para su interpretación.

La segunda fase consistió en la creación de una base de datos en $\mathrm{Excel}^{\circledR}$, la cual estuvo orientada al análisis de la información obtenida a través de App Usage ${ }^{\circledR}$.

Se utilizó la aplicación App Usage $\circledR_{\text {, ya que }}$ cumplía con todos los requisitos establecidos para criterios de esta investigación. La aplicación brindó la siguiente información:

a) Historial del uso de aplicaciones: recolectó datos de cuánto tiempo el usuario utilizó el móvil en cada hora del día.

b) Historial de uso del teléfono: recolectó información de cuántas veces se encendió la pantalla del teléfono en el día.

c) Historial de actividad: recolectó datos de cuánto tiempo el usuario permaneció en una aplicación específica.

d) Principales aplicaciones utilizadas: presentó un resumen de las aplicaciones que se utilizaron con mayor frecuencia en el día.

e) Enviar correo de resumen: envió a las 00:00 horas un resumen completo del historial de uso del teléfono móvil.

Descrita ya la población a estudiar, la hipótesis de investigación fue "los estudiantes utilizan sus dispositivos móviles como una herramienta de distracción más que como una herramienta proactivamente académica".

\section{RESULTADOS}

Las características de la muestra que participó en el estudio fueron: $39.47 \%(n=15)$ varones y $60.53 \%(n=23)$ mujeres. La edad promedio en años de los participantes en estudio fue: moda de 20.5 años (21 años), con un rango entre 20 y 27 años. Los estudiantes utilizan el móvil frecuentemente. (Ver gráfico No. 1).
Gráfico No. 1: Tiempo de uso del móvil de los estudiantes de 4 años de medicina de la EUCS/UNAH-VS.

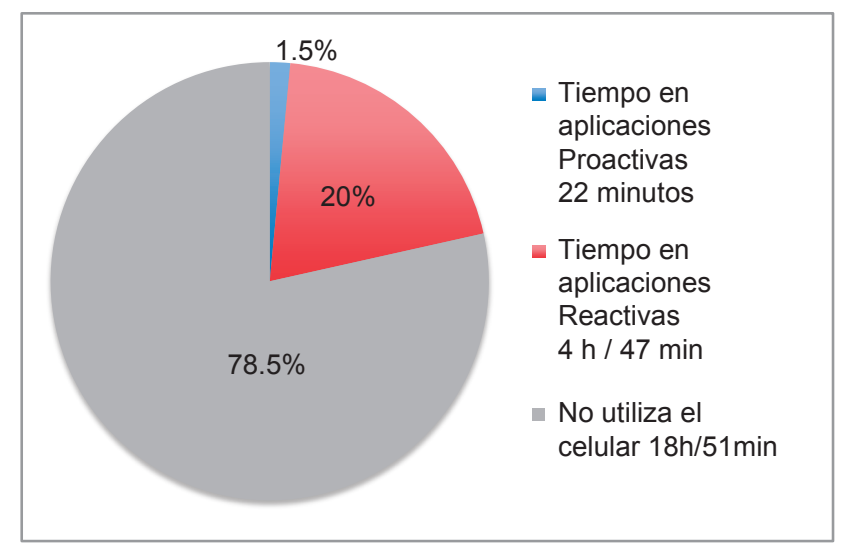

Fuente: Instrumento App Usage.

Los estudiantes utilizan más sus móviles en aplicaciones reactivas, siendo la mas usada el Whatsapp. (Ver tabla No. 1).

Tabla No. 1: Las aplicaciones reactivas y proactivas que utilizan más los estudiantes de la EUCS/UNAH-VS.

\begin{tabular}{|lll|}
\hline $\begin{array}{c}\text { Aplica- } \\
\text { ciones }\end{array}$ & \multicolumn{1}{c|}{$\begin{array}{c}\text { Tipo de } \\
\text { Aplicación }\end{array}$} & $\begin{array}{c}\text { Preferencia } \\
\text { de uso }\end{array}$ \\
\hline Proactivas & Polaris PDF viewer & Primera \\
\hline & Microsoft Office Word & Segunda \\
\hline & Adobe PDF viewer & Tercera \\
\hline & & \\
\hline Reactivas & Whatsapp & Primera \\
\hline & Facebook & Segunda \\
\hline & Instagram & Tercera \\
\hline
\end{tabular}

Fuente: Instrumento App Usage.

Los estudiantes utilizan el celular frecuentemente en las siguientes horas: en primer lugar las 8:00 pm, seguido a las 9:00 pm y por último a las 6:00 pm.

\section{DISCUSIÓN}

Se pudo determinar que el uso del teléfono móvil fue una constante en el día del estudiante de medicina con un promedio de 5 horas al día, datos similares aporta un estudio europeo en donde indican un uso de más de cuatro horas diarias de internet para el $56 \%$ de la muestra de estudiantes universitarios. ${ }^{(15)}$ Se acotó que la práctica médica 
fue inserta en un complejo sistema de relaciones humanas, por lo que es fundamental actuar con un elevado profesionalismo científico-técnico en donde los distractores como el teléfono móvil juegan un papel importante en la relación médico-paciente. ${ }^{(8)}$

Se observó en los estudiantes un marcado uso del teléfono móvil en horas específicas de la noche y menos en el día en las cuales el estudiante de cuarto año A se encuentra en clases, contrario lo que investigaciones como la del Journal of Clinical and Diagnostic Research afirmaron: que el $24.7 \%$ de su población estudiada, utilizaba constantemente el móvil durante clases o durante procedimientos prácticos laborales. ${ }^{(11)}$ Resultados en voluntarios de una investigación que utilizó el software XMobiSense ${ }^{\circledR}$, afirman que las personas que más utilizan su celular a lo largo del día sobreestiman el tiempo que lo utilizan, al contrario de las personas que consideran utilizar poco tiempo el dispositivo, y también subestiman el tiempo de utilización. ${ }^{(16-18)}$

Contrario con los resultados de esta investigación, es el estudio de la compañía Motorola $^{\mathrm{TM}}$ dirigido por la Dra. Nancy Etcoff, el cual se basó en una encuesta a sus usuarios sobre la naturaleza de utilización de su dispositivo móvil, el $49 \%$ de personas de todas las edades reconocieron utilizar el móvil para lectura de libros o revistas de cualquier índole en un día típico de uso(19) datos que difieren en este estudio en donde solo el $7 \%$ utiliza el dispositivo para aplicaciones proactivas. El concepto de distractor dentro del uso y naturaleza del teléfono móvil fue expuesto en diversos estudios que señalaron el efecto negativo que tiene el uso reactivo del mismo por ejemplo en la
Universidad de Colorado se encuentra que el $53 \%$ de los encuestados posee un smartphone, que es usado principalmente para mensajería corta y para e-mail y, en segundo lugar, como un dispositivo para leer noticias y observar videos en internet ${ }^{(20)}$ otro estudio de Futuro Labs, los jóvenes entre 20 y 29 años, rango de edad en el que generalmente se encuentran los estudiantes universitarios (igual a nuestra muestra con rango de 20-27) hacen uso del smartphone, en mayor porcentaje $(85 \%)$, para acceder a redes sociales ${ }^{(21)}$ datos que se asemejan a nuestro estudio en donde el $93 \%$ utiliza en smartohone para aplicaciones reactivas como whatsapp, facebook e instagram.

\section{Conclusión}

La naturaleza de la utilización del teléfono móvil fue de tipo "reactivo" o como "herramienta de distracción", por lo tanto, se comprobó la hipótesis de investigación: "los estudiantes utilizan sus dispositivos móviles como una herramienta de distracción más que como una herramienta proactivamente académica". Se observó también la utilización de redes sociales, que son las aplicaciones más utilizadas y son consideradas "proactivas".

\section{Recomendación}

Impulsar programas educativos sobre el tiempo y naturaleza del uso del teléfono móvil en la población estudiantil, realizar otras investigaciones de tipo correlacional sobre diversos usos del teléfono móvil y su efecto directo e indirecto en el rendimiento académico y crear un instrumento de recolección de información que mejore la consignación de los datos obtenidos a partir de aplicaciones móviles. 


\section{REFERENCIAS BIBLIOGRÁFICAS}

1. Garrote Rojas D, Jimenez Fernandez S, Gomez Barreto I. Problemas Derivados del Uso de Internet y el Teléfono Móvil en Estudiantes Universitarios. Form Univ [internet].2018 [consultado el 13 de marzo de 2018];11(2):99-108. Disponible en: https:// dx.doi.org/10.4067/ S0718-500620180002000 99.

2. Winocur R. Internet en la vida cotidiana de los jóvenes. Rev Mex Sociol [internet]. 2006[consultado el 13 de marzo de 2018]; 68(3): 551-580. Disponible en: http://www.scielo.org.mx/scielo.php? script=sci_arttext\&pid=S0188-2503200 $6000300005 \&$ Ing $=$ es\&tlng $=$ es .

3. Universidad Nacional Autónoma de Honduras. Facultad de Ciencias Médicas. Perfil de la carrera de medicina: plan de estudios medicina [Internet] Tegucigalpa: Facultad de Ciencias Médicas, UNAH: s.f. [Consultado 1 mayo 2018]. Disponible en: https:// www. unah.edu.hn/assets/UNAH/plan-de-es tudios/ PLAN-DE-ESTUDIOS-ME DICI NA-Y-CIRUGIA.pdf.

4. Haug S, Castro RP, Kwon M, Filler A, Kowatsch T Schaub MP. Smartphone use and smartphone addiction among young people in Switzerland. J Behav Addict [Internet]. 2015; 4(4): 299-307.

5. Chatterjee S. A Sociological Outlook of Mobile Phone Use in Society. IJIMS. 2014;1(6):55-63

6. Comisión Nacional de Telecomunicaciones $(\mathrm{HN})$. Desempeño del sector de telecomunicaciones en Honduras. Informe Estadístico. Comayagüela: CONATEL; 2016.

7. Tábora MR. Competencia y regulación en las telecomunicaciones: el caso de Honduras. México: CEPAL; 2007. (Serie estudios y perspectiva, 84).

8. Sánchez MR. Nomofobia y su relación con la adicción a las redes sociales: estudio realizado en la escuela Normal privada y colegio integral sololateco del departamento y municipio de Sololá. [Tesis]. Quetzaltenango: Universidad Rafael Landívar, Facultad de Humanidades; 2013.

9. Prieto SE, Gutiérrez F, Jiménez CA, Méndez $D$. El teléfono celular como distractor de la atención médica en un servicio de urgencias. Med Int Méx. 2013; 29(1):39-47.

10. Villanueva Silvestre V. Programa de prevención del abuso y la dependencia del teléfono móvil en población adolescente. [Tesis doctoral]. Valencia: Universitat de Valencia, Departamento de psicología básica; 2012.

11. Prasad M, Patthi B, Singla A, Gupta R. Nomophobia: A cross-sectional study to assess mobile phone usage among dental students. J Clin Diagn Res. 2017; 11(2):34-39.

12. Gálvez N. Uso del celular y sus efectos en el rendimiento académico. [Tesis doctoral]. Guatemala: Universidad de San Carlos de Guatemala, Escuela de Ciencias de la Comunicación; 2012.

13. Matoza Báez CM, Carballo Ramírez MS. Nivel de Nomofobia en estudiantes de medicina de Paraguay, año 2015. CIMEL. 2016; 21(1) 28-30.

14. App Usage - Manage/Track Usage. In: google play store [Internet]. Hsinchu: Sam Lu; 2011. [actualizado 25 de abril 2018, consultado 18 enero de 2018]. Disponible en: https://play.google.com/ store/apps/details?id=com.a0soft.gpho ne.uninstaller\&hl=en. 
15. Garmendia M, Garitaonandia C, Martínez G. Casado M A. Riesgos y segu ridad en Internet: Los menores españoles en el contexto europeo. Bilbao: EU Kids Online; 2011.

16. Gonzáles C, Muñoz L. Redes sociales su impacto en la educación superior: Caso estudio Universidad Tecnológica de Panamá. Campus Virtuales. 2016; 5(1): 84-90.

17. Shah J, Hag U, Bashir A, Shah SA. Awareness of academic use of smartphones and medical apps among medical students in a private medical college. J Pak Med Assoc. 2016;66(2):184-6.

18. Goedhart G, van Wel L, Langer C, Viladoms P, Wiart J, Hours M, et al. Recall of mobile phone usage and laterality in young people: The multinational Mobi-Expo study. Environ Res [internet]. 2018 [consultado el 25 de mayo de 2018]; 165:150-157. Disponible en: https:// www.sciencedirect.com/science/ article/pii/S0013935118302172?via\%3 Dihub.
19. IPSOS. Motorola Phone-Life balance study data report by generations [nternet]. París: IPSOS; 2018. [consultado el 1 de mayo de 2018]. Disponible en: https:// www.ipsos.com/sites/default/files/ ct/news/documents/2018-02/motorola -phone-life-balance-study-topline-2018 $-02-21$.pdf.

20. Dean J. Digital Media Test Kitchen [Internet]. [consultado el 25 de mayo de 2018]. Colorado: DMTK; 2010. Disponible en: http://testkitchen.colorado.edu/reports/ smartphone/smartphone-survey/.

21. Futuro Labs. Jóvenes Millennials: Perfiles, hábitos y actitudes en el uso del Smartphone, en Lima 2014 [Internet].Lima: Futuro Labs; 2014. [consultado el 25 de mayo de 2018]. Disponible en: http://www.futurolabs.com/los-millen nials-de-lima-y-el-uso-del-smartphone/\# prettyPhoto. 